\title{
ABDELLATIF ZEGGAR \\ Nombre de Lefschetz basique pour un feuilletage riemannien
}

\author{
Annales de la faculté des sciences de Toulouse $\sigma^{e}$ série, tome $1, \mathrm{n}^{\circ} 1$ \\ (1992), p. 105-131 \\ <http://www.numdam.org/item?id=AFST_1992_6_1_1_105_0>
}

(C) Université Paul Sabatier, 1992, tous droits réservés.

L'accès aux archives de la revue «Annales de la faculté des sciences de Toulouse » (http://picard.ups-tlse.fr/ annales/) implique l'accord avec les conditions générales d'utilisation (http://www.numdam.org/conditions). Toute utilisation commerciale ou impression systématique est constitutive d'une infraction pénale. Toute copie ou impression de ce fichier doit contenir la présente mention de copyright.

\section{NumDam}

Article numérisé dans le cadre du programme Numérisation de documents anciens mathématiques http://www.numdam.org/ 


\title{
Nombre de Lefschetz basique pour un feuilletage riemannien
}

\author{
AbDellatif ZegGar ${ }^{(1)}$
}

\begin{abstract}
RÉSUMÉ. - En adaptant les techniques de Toledo [To] on établit une formule intégrale donnant le nombre de Lefschetz basique pour un endomorphisme géométrique d'un complexe transversalement elliptique associé à une application préservant un feuilletage riemannien sur une variété compacte.
\end{abstract}

Abstract. - By adapting the techniques of Toledo [To] we establish an integral formula giving the basic Lefschetz number for a geometric endomorphism of transversally elliptic complex, associated to a map preserving a Riemannian foliation on a compact manifold.

\section{Introduction}

Un difféomorphisme $f$ d'une $m$-variété compacte $M$ induit pour chaque $i=0, \ldots, \operatorname{dim} M$ un isomorphisme $H^{i}(f)$ au niveau de chaque espace vectoriel de cohomologie à coefficients complexes $H^{i}(M)$ qui est de dimension finie. Le nombre $L(f)=\sum_{i}(-1)^{i} \operatorname{Tr} H^{i}(f)$ (où $\operatorname{Tr} H^{i}(f)$ est la trace de $\left.H^{i}(f)\right)$ est appelé le nombre de Lefschetz de $f$. La condition $L(f)=0$ est une condition nécessaire pour que la transformation $f$ soit sans point fixe. Atiyah et Bott $[\mathrm{AB}]$ ont montré que ce nombre peut être calculé à l'aide d'invariants (tels que la trace, l'indice,...) de certains opérateurs elliptiques définis sur $M$. La structure différentiable de la variété est exploitée de façon capitale mais il est possible d'étendre ces résultats à certains espaces singuliers. C'est l'objet de ce travail.

(1) U.R.A. au CNRS 751, Université de Valenciennes, Le Mont-Houy, 59326 Valenciennes Cedex (France) 
Nous commençons d'abord par établir une formule de Lefschetz invariante par l'action d'un groupe de Lie compact sur une variété compacte connexe; ensuite nous appliquons le résultat obtenu pour donner une formule intégrale calculant le nombre de Lefschetz basique d'un endomorphisme géométrique $T$ d'un complexe transversalement elliptique à un feuilletage riemannien minimalisable $(M, \mathcal{F})$. Nous obtenons en quelque sorte une formule de Lefschetz sur l'espace des adhérences des feuilles qui est un espace singulier. Goresky et Macpherson ont démontré un résultat analogue sur un espace singulier muni d'une stratification mais avec des méthodes différentes. Celles que nous utilisons sont inspirées des techniques de Toledo [To]. Le cas particulier de l'endomorphisme identité du complexe $\Omega^{*}(M / \mathcal{F})$ des formes basiques complexes, nous a permis d'introduire la classe d'Euler basique qui généralise la notion classique de classe d'Euler.

Dans la section 1 nous rappellons la définition d'un feuilletage riemannien et le théorème de strucutre de Molino ainsi que la notion de $\mathcal{F}$-fibré hermitien, de section basique et d'opérateur différentiel basique transversalement elliptique. Nous résumons d'autre part les résultats sur la décomposition de Hodge pour de tels opérateurs décrite dans [Ek] qui permet de justifier la définition du nombre de Lefschetz basique. Dans la section 2 nous adaptons les techniques de Toledo pour obtenir une formule intégrale donnant le nombre de Lefschetz invariant sous l'action d'un groupe de Lie compact. Ces résultats sont appliqués dans le paragraphe 3 pour établir notre résultat principal, c'est-à-dire une formule intégrale permettant de calculer le nombre de Lefchetz basique d'un endomorphisme géométrique d'un complexe transversalement elliptique à un feuilletage riemannien.

Toutes les structures considérées seront de classe $C^{\infty}$.

\section{Quelques notations, rappels et définitions}

Soient $M$ une variété compacte connexe orientée munie d'un feuilletage $\mathcal{F}$ défini par un recouvrement ouvert $\left(U_{i}\right)_{i \in I}$ de $M$, une variété $T$ de dimension $q$ (appelée variété transverse) et des submersions : $U_{i} \stackrel{f_{i}}{\longrightarrow} T$ à fibres connexes compatibles dans le sens suivant : pour $i, j \in I$ tels que $U_{i} \cap U_{j} \neq \emptyset$, il existe un difféomorphisme $\gamma_{i j}$ de l'ouvert $f_{j}\left(U_{i} \cap U_{j}\right)$ sur l'ouvert $f_{i}\left(U_{i} \cap U_{j}\right)$ tel que $f_{i}=\gamma_{i j} \circ f_{j}$; les feuilles de la restriction de $\mathcal{F}$ à $U_{i}$ sont les fibres de $f_{i}$. On désignera par $T \mathcal{F}$ le fibré tangent à $\mathcal{F}$ et $\nu \mathcal{F}=T M / T \mathcal{F}$ son fibré normal; $\chi(M)$ et $\Gamma(\mathcal{F})$ sont les modules des sections respectivement de $T M$ et $T \mathcal{F}$ sur l'anneau $C^{\infty}(M)$ des fonctions de classe $C^{\infty}$ sur $M$. 
Une forme différentielle complexe $\alpha$ sur $M$ est dite basique si pour tout $X$ dans $\Gamma(\mathcal{F})$, la dérivée de Lie $L_{X} \alpha$ et le produit intérieur $i_{X} \alpha$ sont nuls. Les formes basiques constituent une sous-algèbre différentielle $\Omega^{*}(M / \mathcal{F})$ de l'algèbre $\Omega^{*}(M)$ des formes différentielles complexes sur $M$; la cohomologie de $\left(\Omega^{*}(M / \mathcal{F}), d\right)$ est appelée la cohomologie basique de $M$ et est notée $H^{*}(M / \mathcal{F})$. Un champ de vecteurs $Y$ sur $M$ est dit feuilleté si $[X, Y] \in \Gamma(\mathcal{F})$ pour tout $X$ dans $\Gamma(\mathcal{F})$; c'est-à-dire $Y$ est un automorphisme infinitésimal de $\mathcal{F}$. On vérifie aisément que $Y$ est feuilleté si et seulement si le groupe à un paramètre engendré par $Y$ respecte $\mathcal{F}$. L'espace $\Gamma(\mathcal{F})$ est un idéal de l'algèbre des champs feuilletés $\chi(M, \mathcal{F})$ et l'algèbre de Lie quotient

$$
\chi(M / \mathcal{F})=\chi(M, \mathcal{F}) / \Gamma(\mathcal{F})
$$

est appelée l'algèbre de Lie des champs basiques. La classe d'équivalence dans $\chi(M / \mathcal{F})$ d'un champ feuilleté $Y \in \chi(M, \mathcal{F})$ sera notée $Y_{b}$.

On dira qu'un $q$-uple $\left(Y^{1}, \ldots, Y^{q}\right)$ de champs feuilletés définit un $p a$ rallélisme transverse pour $\mathcal{F}$ si le $q$-uple $\left(Y_{b}^{1}, \ldots, Y_{b}^{q}\right)$ des champs basiques associés est de rang $q$ en tout point (et donc trivialise le fibré $\nu \mathcal{F}$ ). Si un tel parallélisme existe, on dit que $\mathcal{F}$ est transversalement parallélisable (T.P. en abrégé). Dans le cas particulier où le sous-espace vectoriel de $\chi(M / \mathcal{F})$ engendré par $\left(Y_{b}^{1}, \ldots, Y_{b}^{q}\right)$ est une sous-algèbre de Lie $\mathcal{G}$, on dit que $\mathcal{F}$ est un $\mathcal{G}$-feuilletage de Lie. Un parallélisme transverse permet de munir le fibré normal d'un feuilletage T.P. d'une structure riemanienne invariante le long des feuilles.

De manière générale on dira que $\mathcal{F}$ est riemannien s'il peut être défini par des submersions locales $U_{i} \stackrel{f_{i}}{\longrightarrow} T$ au-dessus d'une variété riemannienne $T$ telles que les difféomorphismes de transition $\gamma_{i j}$ sont des isométries locales de $T$. Pour un tel feuilletage, $\nu \mathcal{F}$ est muni d'une métrique riemannienne invariante le long des feuilles qu'on pourra compléter en une métrique riemannienne sur $M$ dont on dira qu'elle est quasi-fibrée. Pour étudier la structure de ce feuilletage, on peut supposer, quitte à passer à un revêtement à deux feuillets, qu'il est transversalement orienté, c'est-à-dire, $\nu \mathcal{F}$ est orienté. Soit alors $M^{\sharp}$ le $\mathrm{SO}(q)$-fibré principal des repères orthonormés directs de $\nu \mathcal{F}$. On a le théorème de structure dû à P. Molino [Mo].

THÉORÈME 1.1. - Soit $\mathcal{F}$ un feuilletage riemannien transversalement orienté de codimension $q$ sur une variéte compacte connexe $M$.

Si $\mathcal{F}$ est T.P., alors 
i) les adhérences des feuilles de $\mathcal{F}$ sont les fibres d'une fibration localement triviale $F \longrightarrow M \stackrel{\pi}{\rightarrow} W$ appelée fibration basique de $\mathcal{F}$;

ii) il existe une algèbre de Lie $\mathcal{G}$ et un $\mathcal{G}$-feuilletage de Lie $\left(F, \mathcal{F}_{0}\right) \dot{a}$ feuilles denses tel que le feuilletage induit sur chaque fibre de $\pi$ est isomorphe à $\left(F, \mathcal{F}_{0}\right)$;

iii) le groupe structural de la fibration basique est un sous-groupe $d u$ groupe $\operatorname{Diff}\left(F, \mathcal{F}_{0}\right)$ des difféomorphismes de $F$ qui respectent $\mathcal{F}_{0}$.

Si $\mathcal{F}$ n'est pas nécessairement T.P., alors

iv) il se relève en un feuilletage T.P. de même dimension, $\mathcal{F}^{\sharp}$ sur $M^{\sharp}$ invariant par l'action de $\mathrm{SO}(q)$.

\section{2. $\mathcal{F}$-fibrés hermitiens}

Rappelons qu'une connexion sur un fibré vectoriel complexe $E$ au-dessus de $M$ est une application C-linéaire $\nabla: \Gamma(E) \longrightarrow \operatorname{Hom}(T M, E)$ vérifiant

$$
\nabla(f s)=d f \otimes s+f \nabla(s), \quad \forall f \in C^{\infty}(M), \forall s \in \Gamma(E) .
$$

Le morphisme d'espaces vectoriels qui à un champ de vecteurs $X$ sur $M$ associe l'élément $\nabla_{X}$ de $\operatorname{End}(\Gamma(E))$ défini par :

$$
\nabla_{X}(s)=(\nabla s)(X), \quad \forall s \in \Gamma(E)
$$

n'est pas un morphisme d'algèbres de Lie en général; ceci est mesuré par la courbure $R$ de $\nabla$ qui est une application bilinéaire altérnée

$$
R: \chi(M) \times \chi(M) \longrightarrow \operatorname{End}(\Gamma(E))
$$

définie par :

$$
R(X, Y)=\left[\nabla_{X}, \nabla_{Y}\right]-\nabla_{[X, Y]}=\nabla_{X} \nabla_{Y}-\nabla_{Y} \nabla_{X}-\nabla_{[X, Y]} .
$$

Une connexion $\nabla$ sur $E$ est dite basique (cf. [KT]) si sa courbure $R$ vérifie

$$
i_{X} R=0, \quad \forall X \in \Gamma(\mathcal{F})
$$


Un fibré vectoriel complexe $E$ sur $M$ muni d'une connexion basique $\nabla$ sera appelé un $\mathcal{F}$-fibré; une section s de $E$ vérifiant

$$
\nabla_{X}(s)=0, \quad \forall X \in \Gamma(\mathcal{F})
$$

sera dite basique.

L'ensemble $\Gamma(E / \mathcal{F})$ des sections basiques de $E$ est un module sur l'anneau $C^{\infty}(M / \mathcal{F})$ des fonctions basiques $\operatorname{sur}(M, \mathcal{F})$.

On considère maintenant $\left(E, \nabla^{E}\right)$ et $\left(F, \nabla^{F}\right)$ deux $\mathcal{F}$-fibrés sur $M$ et $\varphi: E \longrightarrow F$ un morphisme de fibrés vectoriels induisant l'identité sur $M$.

On dira que $\varphi$ est un morphisme de $\mathcal{F}$-fibrés si

$$
\nabla_{X}^{F} \varphi(s)=\varphi\left(\nabla_{X}^{E} s\right), \quad \forall X \in \Gamma(\mathcal{F}), \forall s \in \Gamma(E) .
$$

Il est clair que les morphismes de $\mathcal{F}$-fibrés de $\left(E, \nabla^{E}\right)$ dans $\left(F, \nabla^{F}\right)$ s'identifient naturellement aux sections basiques du $\mathcal{F}$-fibré $(L(E, F) ; \nabla)$ où $\nabla$ est la connexion basique définie par

$$
\left(\nabla_{X} \varphi\right)(s)=\nabla_{X}^{F} \varphi(s)-\varphi\left(\nabla_{X}^{E} s\right)
$$

pour tout $X \in \chi(M), \varphi \in \Gamma(L(E, F))$ et $s \in \Gamma(E)$.

Soit $E$ un fibré vectoriel complexe sur $M$ muni d'une connexion basique $\nabla$ et d'une métrique hermitienne notée $\langle\mid\rangle$; alors $\nabla$ induit d'une manière naturelle une structure de $\mathcal{F}$-fibré sur le fibré vectoriel complexe $\mathcal{S}(E)$ sur $M$ ayant pour fibre en un point $x \in M$ l'espace vectoriel des formes sésquilinéaires sur la fibre $E_{\boldsymbol{x}}$.

On dit que $E$ est un $\mathcal{F}$-fibré hermitien si la métrique hermitienne $\langle\mid\rangle$ est basique en tant que section du $\mathcal{F}$-fibré $\mathcal{S}(E)$.

Par exemple si $\mathcal{F}$ est un feuilletage riemannien, la structure riemannienne et la connexion de Levi-Cevita transverses (cf. [Mo]) sur le fibré normal $\nu \mathcal{F}$ induisent d'une manière naturelle une métrique hermitienne et une connexion basique sur le complexifié $(\nu \mathcal{F})_{\mathbf{C}}$ de $\nu \mathcal{F}$, pour lesquelles $(\nu \mathcal{F})_{\mathbf{C}}$ est un $\mathcal{F}$-fibré hermitien. 


\subsection{Opérateurs transversalement elliptiques}

Considèrons deux $\mathcal{F}$-fibrés $E$ et $F$ sur $(M, \mathcal{F})$ et notons par $\Im_{E}$ le préfaisceau sur $M$ qui à tout ouvert $U \subset M$ associe l'espace $\Gamma\left(E_{U} / \mathcal{F}\right)$ des sections basiques du $\mathcal{F}$-fibré $E_{U}$.

Un opérateur basique d'ordre $m$ de $E$ dans $F$ est un morphisme de préfaisceaux $d: \Im_{E} \longrightarrow \Im_{F}$ noté tout simplement $d: \Gamma(E / \mathcal{F}) \longrightarrow \Gamma(F / \mathcal{F})$ tel que sur tout ouvert $U$ de $M$ distingué pour $\mathcal{F}$ et trivialisant $E$ et $F$ de manière feuilletée, $d_{U}$ s'ecrit sous la forme

$$
d_{U}=\sum_{k=0}^{m} P_{k}\left(\frac{\partial}{\partial y_{1}}, \ldots, \frac{\partial}{\partial y_{q}}\right)
$$

où pour tout $k=0, \ldots, m, P_{k}$ est un polynôme homogène de degré $k$ en $\partial / \partial y_{1}, \ldots, \partial / \partial y_{q}$ dont les coefficients sont des fonctions basiques sur $U$ à valeurs dans l'espace des matrices complexes à $\operatorname{rang}(F)$ lignes et $\operatorname{rang}(E)$ colonnes; $y_{1}, \ldots, y_{q}$ étant les coordonnées transverses à $\mathcal{F}$ sur $U$ (voir [Ek] pour plus de détails).

Soit $d$ un opérateur basique d'ordre $m$ de $E$ dans $F$; pour un point $x \in M$ et un covecteur basique $\xi_{x} \in\left(\nu_{x} \mathcal{F}\right)^{*}$ on définit le symbole principal de $d$ en $\xi_{x}$ comme étant l'application linéaire $\sigma_{d}\left(\xi_{x}\right): E_{x} \longrightarrow F_{x}$ définie pour tout $u_{x} \in E_{x}$ par

$$
\sigma_{d}\left(\xi_{x}\right) \cdot u_{x}=\frac{1}{m !}\left[d_{U}\left(\varphi^{m} s\right)\right](x)
$$

où $d_{U}$ est la restriction de $d$ à un voisinage ouvert $U$ de $x$ distingué pour $\mathcal{F}$ et trivialisant $E$ et $F$ (toujours de manière feuilletée), $\varphi$ est une fonction basique sur $U$ vérifiant $\varphi(x)=0$ et $d \varphi(x)=\xi_{x}$ et $s$ est un élément de $\Gamma\left(E_{U} / \mathcal{F}\right)$ tel que $s(x)=u_{x}$. On montre sans peine que $\sigma_{d}\left(\xi_{x}\right)$ ne dépend pas du choix de $U, \varphi$ et $s$ et que si $\xi_{x}=\sum_{k=1}^{q} \xi_{x}^{k} d y_{k}(x)$, la matrice de $\sigma_{d}\left(\xi_{x}\right)$ par rapport aux repères locaux sur $U$ est égale à $P_{m}\left(\xi_{x}^{1}, \ldots, \xi_{x}^{q}\right)$.

On dit que $d$ est transversalement elliptique, si $\sigma_{d}\left(\xi_{x}\right)$ est un isomorphisme pour tout $\xi_{x}$ non nul dans $\left(\nu_{x} \mathcal{F}\right)^{*}$. Lorsque $E=F$ et $d$ d'ordre pair, on dira que $d$ est fortement transversalement elliptique si pour tout $\xi_{\boldsymbol{x}}$ non nul dans $\left(\nu_{x} \mathcal{F}\right)^{*}, \sigma_{d}\left(\xi_{x}\right)$ est défini positif.

Si $\mathcal{F}$ est le feuilletage par points, la notion d'opérateur différentiel basique transversalement elliptique coïncide avec celle d'opérateur différentiel elliptique au sens usuel. 


\subsection{Décomposition de Hodge basique}

Si $(M, \mathcal{F})$ est T.P. de fibration basique $\pi: M \longrightarrow W$ et $E$ un $\mathcal{F}$ fibré hermitien, pour chaque $w \in W$ on note par $\bar{E}_{w}$ l'espace vectoriel $\Gamma\left(E_{\pi^{-1}(w)} / \mathcal{F}\right)$ des sections basiques du $\mathcal{F}$-fibré hermitien $E_{\pi^{-1}(w)}$ et par $\bar{E}$ l'union disjointe $\bigsqcup_{w \in W} \bar{E}_{w}$. Il a été démontré dans [Ek] que :

i) $\bar{E}$ est un fibré hermitien sur $W$ (appelé fibré utile associé à $E$ ),

ii) On a un isomorphisme naturel de $C^{\infty}(W)$-modules $\Psi_{E}$ de $\Gamma(E / \mathcal{F})$ sur $\Gamma(\bar{E})$ défini par

$$
\Psi_{E}(s)(w)=s_{\mid \pi^{-1}(w)} \quad \text { pour } s \in \Gamma(E / \mathcal{F}) \text { et } w \in W
$$

de plus la mesure canonique sur $M$ induit une mesure sur $W$ qui pérmet de définir un produit scalaire sur $\Gamma(\bar{E})$ pour lequel $\Psi_{E}$ est un isomorphisme d'espaces préhilbertiens,

iii) pour tout opérateur basique

$$
d: \Gamma(E / \mathcal{F}) \longrightarrow \Gamma(F / \mathcal{F}), \quad \bar{d}=\Psi_{E} \circ d \circ \Psi_{F}^{-1}
$$

est un opérateur différentiel de $\bar{E}$ dans $\bar{F}$. De plus, $\bar{d}$ est elliptique si $d$ est transversalement elliptique.

$\mathrm{Si}(M, \mathcal{F})$ est un feuilletage riemannien transversalement orienté, on note toujours $\rho: M^{\sharp} \longrightarrow M$ le $\mathrm{SO}(q)$-fibré principal des repères orthonormés directs de $\nu \mathcal{F}$ et $\mathcal{F}^{\sharp}$ le feuilletage T.P. sur $M^{\sharp}$ relevé de $\mathcal{F}$ dont la fibration basique est notée $M^{\sharp} \longrightarrow W$. Si $E$ est un $\mathcal{F}$-fibré hermitien sur $M$ et $E^{\sharp}$ le fibré sur $M^{\sharp}$ image réciproque de $E$ par $\rho$, on a (cf. [Ek]) :

iv) $E^{\sharp}$ est à la fois un $\mathcal{F}^{\sharp}$-fibré hermitien et un $\operatorname{SO}(q)$-fibré,

v) l'action de $\mathrm{SO}(q)$ sur le fibré $E^{\sharp} \longrightarrow M^{\sharp}$ induit une action sur le fibré utile $\overline{E^{\sharp}} \longrightarrow W$ et en fait un $\mathrm{SO}(q)$-fibré vectoriel muni d'une métrique hermitienne $\mathrm{SO}(q)$-invariante,

vi) l'anneau $C^{\infty}(M / \mathcal{F})$ s'identifie à l'anneau $C_{\mathrm{SO}(q)}^{\infty}(W)$ des fonctions de classe $C^{\infty}$ qui sont constantes sur les orbites de $\operatorname{SO}(q)$ sur $W$ et le $C_{\mathrm{SO}(q)}^{\infty}(W)$-module $\Gamma(E / \mathcal{F})$ est isomorphe aux $C_{\mathrm{SO}(q)}^{\infty}(W)$-modules

$$
\Gamma_{\mathrm{SO}(q)}\left(E^{\sharp} / \mathcal{F}^{\sharp}\right) \quad \text { et } \quad \Gamma_{\mathrm{SO}(q)}\left(\bar{E}^{\sharp}\right)
$$

des éléments $\operatorname{SO}(q)$-invariants respectivement de $\Gamma\left(E^{\sharp} / \mathcal{F}^{\sharp}\right)$ et $\Gamma\left(\overline{E^{\sharp}}\right)$. 
vii) Si $d: \Gamma(E / \mathcal{F}) \longrightarrow \Gamma(F / \mathcal{F})$ est un opérateur basique transversalement elliptique, alors il existe un opérateur basique $\mathrm{SO}(q)$-invariant $d^{\sharp}: \Gamma\left(E^{\sharp} / \mathcal{F}^{\sharp}\right) \longrightarrow \Gamma\left(F^{\sharp} / \mathcal{F}^{\sharp}\right)$ tel que l'opérateur différentiel $\overline{d^{\sharp}}$ est elliptique dans la "direction transverse" aux orbites de $\mathrm{SO}(q)$ sur $W$ et le diagramme

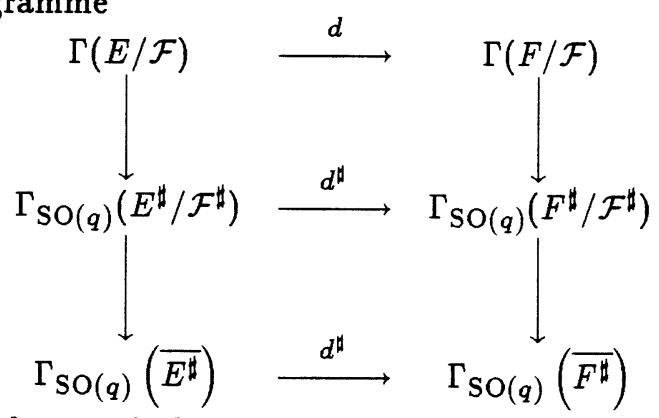

où les flèches verticales sont des isomorphismes, est commutatif. On a le théorème suivant.

THÉORÈME . - Le sous-espace $\operatorname{Ker}(d)$ est de dimension finie et on a une décomposition orthogonale :

$$
\Gamma(E / \mathcal{F})=\operatorname{Ker}(d) \oplus \operatorname{Im}\left(d^{*}\right) .
$$

Voir [Ek] pour la démonstration de ces résultats.

\subsection{Nombre de Lefschetz basique}

Soit $\left(E_{i}\right)_{0 \leq i \leq N}$ une suite de $\mathcal{F}$-fibrés hermitiens sur $M$; supposons donné pour chaque $i=0, \ldots, N-1$ un opérateur basique d'ordre $m$

$$
d_{i}: \Gamma\left(E_{i} / \mathcal{F}\right) \longrightarrow \Gamma\left(E_{i+1} / \mathcal{F}\right) .
$$

Nous supposerons que les $d_{i}$ sont tels que $d_{i+1} d_{i}=0$; ce qui signifie que la suite d'espaces vectoriels et d'applications linéaires $\left(\Gamma\left(E_{i} / \mathcal{F}\right), d_{i}\right)$ définit sur la somme directe de ces espaces une structure d'espace différentiel gradué $(\mathcal{E}, d)$ dont la cohomologie sera notée $H^{*}(\mathcal{E})$.

Pour tout $x \in M$ et pour tout $\xi_{\boldsymbol{x}} \in\left(\nu_{x} \mathcal{F}\right)^{*}$ les symboles principaux

$$
\sigma_{i}\left(\xi_{x}\right): E_{i, x} \longrightarrow E_{i+1, x}
$$

des $d_{i}$ vérifient $\sigma_{i+1}\left(\xi_{x}\right) \circ \sigma_{i}\left(\xi_{x}\right)=0$. Si pour tout $\xi_{x} \neq 0$ la suite d'applications linéaires

$$
0 \longrightarrow E_{0, x} \stackrel{\sigma_{0}\left(\xi_{x}\right)}{\longrightarrow} \ldots \stackrel{\sigma_{N-1}\left(\xi_{x}\right)}{\longrightarrow} E_{N, x} \longrightarrow 0
$$

est exacte, on dira que $(\mathcal{E}, d)$ est un complexe transversalement elliptique $d$ 'ordre $m$ sur $(M, \mathcal{F})$. 
Nombre de Lefschetz basique pour un feuilletage riemannien

Remarquons qu'un complexe transversalement elliptique avec $N=1$ n'est rien d'autre qu'un opérateur transversalement elliptique.

Un des exemples importants de complexes transversalement elliptiques d'ordre un est le complexe $\left(\Omega^{*}(M / \mathcal{F}), d\right)$ des formes basiques sur $M$, qui est formé par les $\mathcal{F}$-fibrés hermitiens $E_{i}=\left(\Lambda^{i} \nu \mathcal{F}\right)_{\mathrm{C}}^{*}$ complexifiés des fibrés des $i$-covecteurs transverses à $\mathcal{F}$.

Si $(\mathcal{E}, d)=\left(\Gamma\left(E_{i} / \mathcal{F}\right), d_{i}\right)_{0<i<N}$ est un complexe transversalement elliptique d'ordre $m$ sur $(M, \mathcal{F})$ alors pour tout $i=0, \ldots, N$ le laplacien

$$
\Delta_{i}=d_{i}^{*} d_{i}+d_{i-1} d_{i-1}^{*}: \Gamma\left(E_{i} / \mathcal{F}\right) \longrightarrow \Gamma\left(E_{i} / \mathcal{F}\right)
$$

est un opérateur basique d'ordre $2 m$ auto-adjoint vérifiant :

i) $\Delta_{i}$ est fortement transversalement elliptique et donc $\operatorname{Ker}\left(\Delta_{i}\right)$ est de dimension finie;

ii) l'application canonique $\operatorname{Ker}\left(\Delta_{i}\right) \longrightarrow H^{i}(\mathcal{E})$ est un isomorphisme et par suite $H^{i}(\mathcal{E})$ est de dimension finie (cf. théorème 1.4).

On peut donc donner la définition suivante.

DÉFINITION .- On appelle nombre de Lefschetz basique d'un endomorphisme $T=\left(T_{i}\right)_{0<i<N}$ de l'espace différentiel gradué $(\mathcal{E}, d)$ le nombre complexe donné par la formule

$$
L_{\mathcal{F}}(T)=\sum_{i=0}^{N}(-1)^{i} \operatorname{Tr} H^{i}(T) .
$$

où $\operatorname{Tr} H^{i}(T)$ désigne la trace de l'application linéaire $H^{i}(T)$ induite par $T$ sur la cohomologie $H^{i}(\mathcal{E})$.

Lorsque $T$ est l'identité $I$ de $(\mathcal{E}, d)$, on a

$$
L_{\mathcal{F}}(I)=\sum_{i=0}^{N}(-1)^{i} \operatorname{dim} H^{i}(\mathcal{E}) ;
$$

qui n'est rien d'autre que la caractéristique d'Euler-Poincaré (ou l'indice) de $(\mathcal{E}, d)$; dans le cas particulier où $N=1,(\mathcal{E}, d)$ est formé d'un seul opérateur transversalement elliptique $d_{0}: \Gamma\left(E_{0} / \mathcal{F}\right) \longrightarrow \Gamma\left(E_{1} / \mathcal{F}\right)$ et on a

$$
L_{\mathcal{F}}(I)=\operatorname{dim} \operatorname{Ker}\left(d_{0}\right)-\operatorname{dim} \operatorname{Coker}\left(d_{0}\right)
$$

c'est l'indice de l'opérateur $d_{0}$. 
Dans ce qui suit nous allons nous intéresser aux nombres de Lefschetz de certains endomorphismes particuliers dits géométriques, qu'on définit de la manière suivante : on se donne $f$ et $\left(\varphi_{i}\right)_{0<i<N}$ où $f: M \longrightarrow M$ est une application qui respecte $\mathcal{F}$ et telle que pour tout point $x$ dans $M$ la différentielle $d f_{x}$ induit un isomorphisme $\nu f_{x}: \nu_{x} \mathcal{F} \longrightarrow \nu_{f(x)} \mathcal{F}$ qui préserve les orientations et pour tout $i=0, \ldots, N, \varphi_{i}: f^{*} E_{i} \longrightarrow E_{i}$ est un morphisme de $\mathcal{F}$-fibrés qui induit l'identité sur la base; puis on définit pour chaque $i=0, \ldots, N$ un endomorphisme $T_{i}$ de $\Gamma\left(E_{i} / \mathcal{F}\right)$ par :

$$
T_{i}(s)(x)=\varphi[x, s \circ f(x)]
$$

$\forall s \in \Gamma\left(E_{i} / \mathcal{F}\right), \forall x \in M$. Si la famille $T=\left(T_{i}\right)_{0<i<N}$ vérifie $d_{i} T_{i}=T_{i+1} d_{i}$ pour tout $i=0, \ldots, N$, on dira que $T$ est un endomorphisme géométrique de $(\mathcal{E}, d)$; pour un tel endomorphisme, l'application $f$ induit une application $f^{\sharp}: M^{\sharp} \longrightarrow M^{\sharp}$ qui à un repère orthonormé direct $z_{x}$ en $x \in M$ associe le repère orthonormé direct $f^{\sharp}\left(z_{x}\right)=\mathcal{N}\left(\nu_{x} f \circ z_{x}\right)$ en $f(x) \in M$ où $\mathcal{N}$ désigne l'orthonormalisation de Schmidt. On vérifie que :

i) $f^{\sharp}$ est une application de classe $C^{\infty}$ qui respecte le feuilletage $\mathcal{F}^{\sharp}$ et qui induit une application de classe $C^{\infty} \bar{f}: W \longrightarrow W$;

ii) les morphismes $\varphi_{i}$ induisent d'une manière naturelle des morphismes

$$
\varphi_{i}^{\sharp}:\left(f^{\sharp}\right)^{*} E_{i}^{\sharp} \longrightarrow E_{i}^{\sharp} \text { et } \overline{\varphi_{i}^{\sharp}}: \bar{f}^{*}\left(\overline{E_{i}^{\sharp}}\right) \longrightarrow \overline{E_{i}^{\sharp}}
$$

tels que les $\varphi_{i}^{\sharp}$ sont des morphismes de $\mathcal{F}^{\sharp}$-fibrés; de plus ces deux familles de morphismes définissent des endomorphismes géométriques $T^{\sharp}$ et $\overline{T^{\sharp}}$ pour les complexes respectifs :

$$
\begin{gathered}
0 \longrightarrow \Gamma\left(E_{0}^{\sharp} / \mathcal{F}^{\sharp}\right) \stackrel{d_{0}^{\sharp}}{\longrightarrow} \ldots \stackrel{\stackrel{d_{N-1}^{\sharp}}{\longrightarrow} \Gamma\left(E_{N}^{\sharp} / \mathcal{F}^{\sharp}\right) \longrightarrow 0}{\longrightarrow} \underset{ }{\longrightarrow} \Gamma\left(\overline{E_{0}^{\sharp}}\right) \stackrel{\overline{d_{0}^{\sharp}}}{\longrightarrow} \ldots \stackrel{\overline{d_{N-1}^{\sharp}}}{\longrightarrow} \Gamma\left(\overline{E_{N}^{\sharp}}\right) \longrightarrow 0
\end{gathered}
$$

qui sont tels que le premier est elliptique dans la "direction horizontale" transverse à $\mathcal{F}^{\sharp}$, et le second est elliptique dans la direction transverse aux orbites de $\mathrm{SO}(q)$ sur $W$.

Le complexe $(\mathcal{E}, d)$ s'identifie au sous-complexe $\left(\overline{\mathcal{E}}_{\mathrm{SO}(q)}, \bar{d}_{\mathrm{SO}(q)}\right)$ des éléments invariants du $\mathrm{SO}(q)$-complexe $\left(\overline{\mathcal{E}^{\sharp}}, \overline{d^{\sharp}}\right)$ sur $W$ formé par les $\operatorname{SO}(q)$ fibrés $\overline{E_{i}^{\sharp}}$ et les opérateurs différentiels invariants $\overline{d_{i}^{\sharp}}$, c'est-à-dire, commutant avec l'action de $\mathrm{SO}(q)$. On est donc amené à étudier le nombre de Lefschetz invariant sur une $G$-variété. 


\section{Nombre de Lefschetz invariant sur une $G$-variété}

Soit $G$ un groupe de Lie compact agissant à droite d'une manière différentiable sur une variété $W$ compacte connexe orientée de dimension $k$. On munit $W$ d'une métrique riemannienne $G$-invariante; la forme volume $\omega$ associée est alors $G$-invariante.

DÉFInITION 2.1. - Une structure de G-fibré, sur un fibré vectoriel complexe $\pi_{E}: E \longrightarrow W$, est la donnée d'une action à droite de $G$ sur $E$ telle que pour tout $g \in G$, on $a$ :

i) $\pi_{E} \circ g=g \circ \pi_{E}$,

ii) pour tout $x \in W, g$ est un isomorphisme linéaire de $E_{x}$ sur $E_{x g}$.

Si $E$ est un $G$-fibré sur $W$, l'action de $G$ sur $E$ induit une action de $G$ sur $\Gamma(E)$ de la manière suivante : pour $g \in G$ et $s \in \Gamma(E)$, on définit $s \cdot g \in \Gamma(E)$ par :

$$
(s \cdot g)(x)=\left[s\left(x \cdot g^{-1}\right)\right] \cdot g, \quad \forall x \in W .
$$

Les sections $s \in \Gamma(E)$ qui vérifient $s \cdot g=s, \forall g \in G$ sont dites invariantes. Leur ensemble est un sous-espace vectoriel de $\Gamma(E)$ noté $\Gamma_{G}(E)$.

Considérons un opérateur différentiel $d: \Gamma(E) \longrightarrow \Gamma(F)$ d'un $G$-fibré $E$ dans un $G$-fibré $F$ sur $W$.

DÉfinition 2.2. - On dit que l'opérateur $d$ est $G$-invariant $s i g \cdot d=d \cdot g$ pour tout $g \in G$.

Un tel opérateur envoie les sections invariantes de $E$ dans les sections invariantes de $F$ et induit donc un opérateur $d_{G}: \Gamma_{G}(E) \longrightarrow \Gamma_{G}(F)$.

Dans toute la suite de ce paragraphe, chaque $G$-fibré $E$ sera muni d'une métrique hermitienne $G$-invariante notée $\langle\quad \mid\rangle$, l'espace vectoriel $\Gamma(E)$ sera alors muni de la structure préhilbertienne définie par le produit scalaire Ginvariant défini pour $\alpha, \beta \in \Gamma(E)$ par

$$
\langle\alpha, \beta\rangle=\int_{W}\langle\alpha(x) \mid \beta(x)\rangle \mathrm{d} x .
$$


THÉORÈME $2.3[\mathrm{Ek}] .-S i d: \Gamma(E) \longrightarrow \Gamma(E)$ est un opérateur elliptique $G$-invariant agissant sur les sections d'un $G$-fibré hermitien $E$ sur $W$, alors l'opérateur $d_{G}: \Gamma_{G}(E) \longrightarrow \Gamma_{G}(E)$ induit pard est tel que :

i) $\operatorname{Ker} d_{G}$ est de dimension finie;

ii) on a la décomposition orthogonale :

$$
\Gamma_{G}(E)=\operatorname{Ker} d_{G} \oplus \operatorname{Im} d_{G}^{*} .
$$

Soit maintenant $(\mathcal{E}, d)=\left\{d_{i}: \Gamma\left(E_{i}\right) \longrightarrow \Gamma\left(E_{i+1}\right)\right\}_{0<i \leq N-1}$ un complexe d'opérateurs différentiels $G$-invariants (supposés d'ordre 1 pour simplifier l'exposé) entre $G$-fibrés hermitiens sur $W$.

DÉFInITION 2.4. - On dira que $(\mathcal{E}, d)$ est transversalement elliptique si pour tout covecteur $\xi_{x} \in T_{x}^{*} W$ non nul et transverse (c'est-à-dire $i_{A} \xi_{x}=0$, $\forall A \in \mathcal{G})$ la suite des symboles

$$
0 \longrightarrow E_{0, x} \stackrel{\sigma_{0}\left(\xi_{x}\right)}{\longrightarrow} \ldots \stackrel{\sigma_{N-1}\left(\xi_{x}\right)}{\longrightarrow} E_{N, x} \longrightarrow 0
$$

est exacte.

Les opérateurs $d_{i, G}: \Gamma_{G}\left(E_{i}\right) \longrightarrow \Gamma_{G}\left(E_{i+1}\right)$ induits par les $d_{i}$ forment un sous-complexe $\left(\mathcal{E}_{G}, d_{G}\right)$ de $(\mathcal{E}, d)$ dont la cohomologie sera notée $H_{G}(\mathcal{E})$ et sera appelée la cohomologie $G$-invariante de $(\mathcal{E}, d)$. Pour les opérateurs laplaciens

$$
\Delta_{i}=d_{i}^{*} d_{i}+d_{i-1} d_{i-1}^{*}: \Gamma\left(E_{i}\right) \longrightarrow \Gamma\left(E_{i}\right),
$$

on a les propriétés suivantes.

i) $\Delta_{i}$ est un opérateur différentiel positif, auto-adjoint et $G$-invariant.

ii) $\Delta_{i}$ est fortement transversalement elliptique, c'est-à-dire, le symbole $\sigma_{\Delta_{i}}\left(\xi_{x}\right)$ de $\Delta_{i}$ en un covecteur transverse $\xi_{x} \in T_{x}^{*} W$ est un automorphisme défini positif de la fibre $E_{i, x}$.

iii) Si $\left(A_{\mu}\right)_{1 \leq \mu \leq \operatorname{dim} G}$ est une base de l'algèbre de Lie $\mathcal{G}$ de $G$ et si pour tout $\mu=1, \ldots, \operatorname{dim} G, L_{A_{\mu}}: \Gamma\left(E_{i}\right) \longrightarrow \Gamma\left(E_{i}\right)$ est l'opérateur différentiel d'ordre 1 défini par :

$$
L_{A_{\mu}}(s)(x)=\left(\frac{\mathrm{d}}{\mathrm{d} t}\right)_{t=0}\left\{s \cdot\left(\exp \left(t A_{\mu}\right)\right)\right\}(x), \quad \forall s \in \Gamma\left(E_{i}\right), \forall x \in W,
$$

alors

$$
Q_{i}=\sum_{\mu=1}^{\operatorname{dim} G} L_{A_{\mu}}^{*} L_{A_{\mu}}
$$


est un opérateur différentiel positif, auto-adjoint, $G$-invariant, nul sur l'espace $\Gamma_{G}\left(E_{i}\right)$, fortement elliptique dans les directions tangentes aux orbites de $G$ sur $W$ et est tel que l'opérateur $D_{i}=\Delta_{i}+Q_{i}$ est fortement elliptique.

iv) En prenant les moyennes sur le groupe $G$ des éléments d'une base orthonormée de vecteurs propres de $D_{i}$ on obtient un système de générateurs pour $\Gamma_{G}\left(E_{i}\right)$ dont on peut extraire une base orthonormée $\left(s_{n}^{i}\right)_{n \in \mathbb{N}}$ avec une suite $\left(\lambda_{n}^{i}\right)_{n \in \mathbb{N}}$ de réels positifs ou nuls vérifiant

$$
\Delta_{i, G}\left(s_{n}^{i}\right)=\lambda_{n}^{i} s_{n}^{i}
$$

Cette décomposition spectrale de $\Delta_{i, G}$ nous permet de définir un opérateur intégral

$$
G_{i}: \Gamma\left(E_{i}\right) \longrightarrow \Gamma\left(E_{i}\right)
$$

par :

$$
G_{i}(s)=\sum_{\lambda_{n}^{i} \neq 0} \frac{1}{\lambda_{n}^{i}}\left\langle s, s_{n}^{i}\right\rangle s_{n}^{i}, \quad \forall s \in \Gamma\left(E_{i}\right)
$$

Notons $K_{i}=d_{i-1}^{*} G_{i}, H_{i}$ la projection orthogonale de $\Gamma\left(E_{i}\right)$ sur $\operatorname{Ker} \Delta_{i, G}$ et $p_{i}$ la projection orthogonale de $\Gamma\left(E_{i}\right)$ sur $\Gamma_{G}\left(E_{i}\right)$ qui n'est rien d'autre que le projecteur défini par :

$$
p_{i}(s)=\int_{G}(s \cdot g) \mathrm{d} g, \quad \forall s \in \Gamma\left(E_{i}\right) .
$$

On a alors le lemme 2.5.

LEMME 2.5

i) $d_{i} G_{i}=G_{i+1} d_{i}$ sur $\Gamma_{G}\left(E_{i}\right)$

ii) $\Delta_{i} G_{i}=G_{i} \Delta_{i}=I-H_{i}$ sur $\Gamma_{G}\left(E_{i}\right)$

et donc

iii) $d_{i-1} K_{i}+K_{i+1} d_{i}=p_{i}-H_{i} \operatorname{sur} \Gamma\left(E_{i}\right)$. 


\section{Abdellatif Zeggar}

\section{Démonstration}

i) Soit $s \in \Gamma_{G}\left(E_{i}\right)$;

si $\Delta_{i} s=0$ alors $d_{i} G_{i}(s)=0=G_{i+1} d_{i}(s)$;

si $\Delta_{i}(s)=\lambda s$ avec $\lambda \neq 0$ alors $s=\sum_{\lambda_{n}^{i}=\lambda}\left\langle s, s_{n}^{i}\right\rangle s_{n}^{i}$ et donc

$$
\begin{aligned}
d_{i} G_{i}(s) & =\sum_{\lambda_{n}^{i}=\lambda} \frac{1}{\lambda}\left\langle s, s_{n}^{i}\right\rangle d_{i} s_{n}^{i} \\
& =\sum_{\lambda_{n}^{i}=\lambda} \sum_{\lambda_{m}^{i+1}=\lambda} \frac{1}{\lambda}\left\langle s, s_{n}^{i}\right\rangle\left\langle d_{i} s_{n}^{i}, s_{m}^{i+1}\right\rangle s_{m}^{i+1} .
\end{aligned}
$$

D'autre part

$$
\begin{aligned}
G_{i+1} d_{i}(s) & =G_{i+1}\left(\sum_{\lambda_{n}^{i}=\lambda}\left\langle s, s_{n}^{i}\right\rangle d_{i} s_{n}^{i}\right) \\
& =G_{i+1}\left(\sum_{\lambda_{n}^{i}=\lambda} \sum_{\lambda_{m}^{i+1}=\lambda}\left\langle s, s_{n}^{i}\right\rangle\left\langle d_{i} s_{n}^{i}, s_{m}^{i+1}\right\rangle s_{m}^{i+1}\right) \\
& =\sum_{\lambda_{n}^{i}=\lambda} \sum_{\lambda_{m}^{i+1}=\lambda} \frac{1}{\lambda}\left\langle s, s_{n}^{i}\right\rangle\left\langle d_{i} s_{n}^{i}, s_{m}^{i+1}\right\rangle s_{m}^{i+1} .
\end{aligned}
$$

Ce qui prouve qu'on a bien $d_{i} G_{i}=G_{i+1} d_{i}$ sur $\Gamma_{G}\left(E_{i}\right)$.

ii) Soit $s \in \Gamma_{G}\left(E_{i}\right)$; on a

$$
\begin{aligned}
\Delta_{i} G_{i}(s) & =\sum_{\lambda_{n}^{i} \neq 0} \frac{1}{\lambda_{n}^{i}}\left\langle s, s_{n}^{i}\right\rangle \Delta_{i}\left(s_{n}^{i}\right) \\
& =\sum_{\lambda_{n}^{i} \neq 0}\left\langle s, s_{n}^{i}\right\rangle s_{n}^{i} \\
& =s-H_{i}(s) ; \\
G_{i} \Delta_{i}(s) & =\sum_{\lambda_{n}^{i} \neq 0} \frac{1}{\lambda_{n}^{i}}\left\langle\Delta_{i}(s), s_{n}^{i}\right\rangle s_{n}^{i} \\
& =\sum_{\lambda_{n}^{i} \neq 0} \frac{1}{\lambda_{n}^{i}}\left\langle s, \Delta_{i}\left(s_{n}^{i}\right)\right\rangle s_{n}^{i} \\
& =s-H_{i}(s) .
\end{aligned}
$$


iii) On a pour tout $s \in \Gamma\left(E_{i}\right)$

$$
\begin{aligned}
d_{i-1} K_{i}(s) & =d_{i-1} d_{i-1}^{*} G_{i}(s) \\
& =d_{i-1} d_{i-1}^{*} G_{i}\left(p_{i} s\right)
\end{aligned}
$$

et

$$
\begin{aligned}
K_{i+1} d_{i}(s) & =d_{i}^{*} G_{i+1} d_{i}\left(p_{i} s\right) \\
& =d_{i}^{*} d_{i} G_{i}\left(p_{i} s\right)
\end{aligned}
$$

d'aprés (i); d'où

$$
\begin{aligned}
d_{i-1} K_{i}(s)+K_{i+1} d_{i}(s) & =\Delta_{i} G_{i}\left(p_{i} s\right) \\
& =p_{i}(s)-H_{i}(s)
\end{aligned}
$$

d'aprés (ii) .

Dans la suite, si $E$ et $F$ sont des fibrés hermitiens sur $W$ et $d$ un opérateur différentiel de $E$ dans $F$, on notera :

- $E^{\prime}$ le fibré $E^{*} \otimes\left(\Lambda^{k} T^{*} W\right)_{\mathbf{C}}$ sur $W$ produit tensoriel du dual de $E$ avec le complexifié du fibré des $k$-covecteurs de $W$.

$-E \otimes F$ le fibré $\pi_{1}^{*} E \otimes \pi_{2}^{*} F$ sur $W \times W$ produit tensoriel externe de $E$ avec $F\left(\pi_{1}\right.$ et $\pi_{2}$ étant la première et la deuxième projection de $W \times W$ sur $W$ ).

- * l'isomorphisme de fibrés vectoriels défini de $E$ sur $E^{\prime}$ par

$$
* u_{x}=u_{x}^{*} \otimes \omega(x), \quad \forall x \in W, \forall u_{x} \in E_{x}
$$

( $u_{x}^{*}$ étant l'image de $u_{x}$ par l'isomorphisme naturel de $E$ sur $E^{*}$ ).

- tr le morphisme de fibrés vectoriels défini de $E \otimes E^{\prime}$ dans $\left(\Lambda^{k} T^{*} W\right)_{\mathbf{C}}$ par

$\operatorname{tr}\left(u_{x} \otimes v_{x}\right)=\left\langle u_{x} \mid *^{-1} v_{x}\right\rangle \omega(x), \quad \forall x \in W, \forall u_{x} \in E_{x}, \forall v_{x} \in E_{x}^{\prime}$.

$-\langle;\rangle: \Gamma(E) \times \Gamma\left(E^{\prime}\right) \longrightarrow \mathbf{C}$ l'accouplement défini par

$$
\langle s, t\rangle=\int_{W} \operatorname{tr}(s \otimes t) .
$$

$-d^{\prime}: \Gamma\left(F^{\prime}\right) \longrightarrow \Gamma\left(E^{\prime}\right)$ l'opérateur différentiel défini par la relation

$$
\langle d s, t\rangle=\left\langle s, d^{\prime} t\right\rangle
$$

pour tout $s \in \Gamma(E)$ et tout $t \in \Gamma\left(F^{\prime}\right)$. 
La densité de $\Gamma\left(E_{i}\right) \otimes \Gamma\left(E_{i+1}^{\prime}\right)$ dans $\Gamma\left(E_{i} \otimes E_{i+1}^{\prime}\right)$ permet de définir des opérateurs

$$
\begin{gathered}
\delta_{i}: \Gamma\left(E_{i} \otimes E_{i+1}^{\prime}\right) \longrightarrow \Gamma\left(E_{i+1} \otimes E_{i+1}^{\prime}\right) \\
\delta_{i}^{\prime}: \Gamma\left(E_{i} \otimes E_{i+1}^{\prime}\right) \longrightarrow \Gamma\left(E_{i} \otimes E_{i}^{\prime}\right)
\end{gathered}
$$

par : $\delta_{i}(s \otimes t)=d_{i} s \otimes t$ et $\delta_{i}^{\prime}(s \otimes t)=s \otimes d_{i}^{\prime} t$ pour tout $s \in \Gamma\left(E_{i}\right)$ et tout $t \in \Gamma\left(E_{i+1}^{\prime}\right)$.

Pour $s \in \Gamma\left(E_{i}\right)$ et $x \in W$, on a

$$
H_{i}(s)(x)=\int_{W} h_{i}(x, y) \cdot s(y) \mathrm{d} y
$$

et

$$
K_{i}(s)(x)=\int_{W} k_{i}(x, y) \cdot s(y) \mathrm{d} y
$$

où $h_{i}$ est la section $C^{\infty}$ de $E_{i} \otimes E_{i}^{\prime}$ donnée par

$$
h_{i}(x, y)=\sum_{\lambda_{n}^{i}=0} s_{n}^{i}(x) \otimes * s_{n}^{i}(y)
$$

et $k_{i}$ est la section $C^{\infty}$ de $E_{i-1} \otimes E_{i}^{\prime}$ définie sur l'ouvert

$$
W \times W-\{(x, y) \in W \times W \mid y \in G(x)\}
$$

par :

$$
k_{i}(x, y)=\sum_{\lambda_{n}^{i} \neq 0} \frac{1}{\lambda_{n}^{i}} d_{i-1}^{*} s_{n}^{i}(x) \otimes * s_{n}^{i}(y)
$$

Les sections $h_{i}$ et $k_{i}$ sont respectivement les noyaux de Schwartz des opérateurs intégraux $H_{i}$ et $K_{i}$; le lien entre ces noyaux est donné par les relations

$$
\delta_{i-1} k_{i}(x, y)+\delta_{i}^{\prime} k_{i+1}(x, y)=-h_{i}(x, y)
$$

qui sont vérifiées pour tout couple $(x, y) \in W \times W$ tel que $x \notin G(y)$ (cf. lemme 2.8). 
Considèrons maintenant des champs $X_{1}, \ldots, X_{l}$ et des 1 -formes $\xi_{1}, \ldots, \xi_{l}$ sur $W$ tels que pour toute 1 -forme $\xi \in \Omega^{1}(W)$ on ait :

$$
\xi=\sum_{j=1}^{l} \xi\left(X_{j}\right) \xi_{j}
$$

(on les construit sans peine à l'aide d'un système de coordonnées locales et d'une partition de l'unité sur $W$ ).

Lemme 2.6 [To]. - Pour toute section $\alpha$ de $E_{i-1} \otimes E_{i}^{\prime}$ au-dessus d'un ouvert $V \subset W \times W$, on a

$$
\operatorname{tr} \Delta^{*} \delta_{i-1}(\alpha)-\operatorname{tr} \Delta^{*} \delta_{i-1}^{\prime}(\alpha)=d \sum_{j=1}^{l} i_{X_{j}} \operatorname{tr} \Delta^{*} \sigma_{i-1}\left(\xi_{j}\right) \otimes I(\alpha)
$$

sur $\Delta^{-1}(V), \Delta: W \longrightarrow W \times W$ étant l'application diagonale.

Soient $(\mathcal{E}, d)=\left(\Gamma\left(E_{i}\right), d_{i}\right)_{0 \leq i \leq N}$ un $G$-complexe transversalement elliptique sur $W$ et $T=\left(T_{i}\right)_{0 \leq i \leq N}$ une famille d'applications linéaires

$$
T_{i}: \Gamma\left(E_{i}\right) \longrightarrow \Gamma\left(E_{i}\right)
$$

telles que $d_{i} T_{i}=T_{i+1} d_{i}$ pour tout $i=0,1, \ldots, N$, alors la famille d'applications linéaires

$$
T_{i, G}: \Gamma_{G}\left(E_{i}\right) \longrightarrow \Gamma_{G}\left(E_{i}\right)
$$

définies par :

$$
T_{i, G}(s)=p_{i} \circ T_{i}(s)=\int_{G} T_{i}(s) \cdot g \mathrm{~d} g, \quad \forall s \in \Gamma_{G}\left(E_{i}\right)
$$

forment un endomorphisme $T_{G}:\left(\mathcal{E}_{G}, d_{G}\right) \rightarrow\left(\mathcal{E}_{G}, d_{G}\right)$ de l'espace différentiel gradué $\left(\mathcal{E}_{G}, d_{G}\right)=\left(\Gamma_{G}\left(E_{i}\right), d_{i, G}\right)_{0 \leq i \leq N}$ et on a donc pour chaque $i=$ $0,1, \ldots, N$ un endomorphisme

$$
H_{G}^{i}(T): H_{G}^{i}(\mathcal{E}) \longrightarrow H_{G}^{i}(\mathcal{E})
$$

de l'espace vectoriel de dimension finie $H_{G}^{i}(\mathcal{E})$. On peut donc donner la définition suivante. 


\section{Abdellatif Zeggar}

DÉfinition 2.7. - On appelle nombre de Lefschetz G-invariant de $T$ le nombre

$$
L_{G}(T)=\sum_{i=0}^{N}(-1)^{i} \operatorname{Tr} H_{G}^{i}(T) .
$$

Remarquons que lorsque $T$ est l'identité $I$ de $(\mathcal{E}, d)$

$$
L_{G}(I)=\sum_{i=0}^{N}(-1)^{i} \operatorname{dim} H_{G}^{i}(\mathcal{E})
$$

est la caractéristique d'Euler-Poincaré du complexe différentiel $\left(\mathcal{E}_{G}, d_{G}\right)$ et si en plus $N=1,(\mathcal{E}, d)$ est formé d'un seul opérateur $G$-invariant $d_{0}$ de $\Gamma\left(E_{0}\right)$ dans $\Gamma\left(E_{1}\right)$ induisant un opérateur de Fredholm (cf. [At])

$$
d_{0, G}: \Gamma_{G}\left(E_{0}\right) \longrightarrow \Gamma_{G}\left(E_{1}\right),
$$

on a

$$
L_{G}(I)=\operatorname{dim} \operatorname{Ker} d_{0, G}-\operatorname{dim} \operatorname{Coker} d_{0, G}
$$

qui n'est autre que l'indice de l'opérateur $d_{0, G}$.

Dorénavant on s'intéressera au cas où l'endomorphisme $T$ est géométrique, c'est-à-dire défini de la manière suivante : on se donne une application de classe $C^{\infty}$

$$
f: W \longrightarrow W
$$

et une famille de morphismes de fibrés vectoriels

$$
\varphi_{i}: f^{*} E_{i} \longrightarrow E_{i}
$$

induisant l'identité sur $W$, puis on définit les $T_{i}$ par :

$$
T_{i}(s)(x)=\varphi_{i}[x, s \circ f(x)], \quad \forall s \in \Gamma\left(E_{i}\right), \forall x \in W .
$$

Pour un tel endomorphisme, nous avons le lemme 2.8 .

Lemme 2.8. - Pour tout couple $(x, y) \in W \times W$ tel que $f(x) \notin G(y)$, on $a$

$$
\delta_{i-1} T_{i-1} \otimes I k_{i}(x, y)+\delta_{i}^{\prime} T_{i} \otimes I k_{i+1}(x, y)=-T_{i} \otimes I h_{i}(x, y)
$$

où I désigne l'endomorphisme identité . 
Démonstration. - Soit $U$ un voisinage $G$-saturé de $y$ ne contenant pas $f(x)$ et soit $s$ une séction de $E_{i}$ à support dans $U$; on a (cf. lemme 2.5)

$$
d_{i-1} K_{i}(s)+K_{i+1} d_{i}(s)=p_{i}(s)-H_{i}(s) ;
$$

donc

$$
d_{i-1} T_{i-1} K_{i}(s)(x)+T_{i} K_{i+1} d_{i}(s)(x)=T_{i} p_{i}(s)(x)-T_{i} H_{i}(s)(x),
$$

soit

$$
\begin{array}{r}
\int_{W} \delta_{i-1} T_{i-1} \otimes I k_{i}(x, z) \cdot s(z) \mathrm{d} z+\int_{W} \delta_{i}^{\prime} T_{i} \otimes I k_{i+1}(x, z) \cdot s(z) \mathrm{d} z \\
=\varphi_{i, x}\left[\int_{G} s\left(f(x) \cdot g^{-1}\right) \cdot g \mathrm{~d} g\right]-\int_{W} T_{i} \otimes I h_{i}(x, z) \cdot s(z) \mathrm{d} z
\end{array}
$$

ou encore, puisque $s\left(f(x) g^{-1}\right)=0, \forall g \in G($ car $\forall g \in G, f(x) \cdot g \notin U)$,

$$
\begin{gathered}
\int_{W} \delta_{i-1} T_{i-1} \otimes I k_{i}(x, z) \cdot s(z) \mathrm{d} z+\int_{W} \delta_{i}^{\prime} T_{i} \otimes I k_{i+1}(x, z) \cdot s(z) \mathrm{d} z \\
=-\int_{W} T_{i} \otimes I h_{i}(x, z) \cdot s(z) \mathrm{d} z .
\end{gathered}
$$

Ceci étant vrai pour toute section $s$ à support dans $U$; on a

$$
\delta_{i-1} T_{i-1} \otimes I k_{i}(x, y)+\delta_{i}^{\prime} T_{i} \otimes I k_{i+1}(x, y)=-T_{i} \otimes I h_{i}(x, y) .
$$

Si $C(f, G)$ désigne l'ensemble des points de coïncidence de $f$ avec $G$, c'està-dire l'ensemble des points $x \in W$ tels que $x$ est un point de coïncidence de $f$ avec un élément de $G$ :

$$
C(f, G)=\{x \in W \mid \exists g \in G: f(x)=g(x)\},
$$

on a le théorème 2.9 .

THÉORÈME 2.9. - Il existe une forme différentielle

$$
\bar{\Psi} \in \Omega^{k-1}(W-C(f, G)),
$$

telle que $d \bar{\Psi}$ se prolonge en une forme globale $\bar{\Phi} \in \Omega^{k}(W)$ vérifiant

$$
\int_{W} \bar{\Phi}=L_{G}(T)
$$




\section{Abdellatif Zeggar}

Démonstration. - Pour tout couple $(x, y) \in W \times W$ tel que $f(x) \notin G(y)$, nous avons (cf. lemme 2.8) :

$$
-T_{i} \otimes I h_{i}(x, y)=\delta_{i-1} T_{i-1} \otimes I k_{i}(x, y)+\delta_{i}^{\prime} T_{i} \otimes I k_{i+1}(x, y)
$$

donc pour $x \in W$ tel que $f(x) \notin G(x)$ on a

$$
\begin{aligned}
&-\sum_{i=0}^{N}(-1)^{i} \operatorname{tr} \Delta^{*} T_{i} \otimes I h_{i}(x)= \sum_{i=0}^{N}(-1)^{i} \operatorname{tr} \Delta^{*} \delta_{i-1} T_{i-1} \otimes I k_{i}(x) \\
&+\sum_{i=0}^{N}(-1)^{i} \operatorname{tr} \Delta^{*} \delta_{i}^{\prime} T_{i} \otimes I k_{i+1}(x) \\
&= \sum_{i=1}^{N}(-1)^{i} \operatorname{tr} \Delta^{*} \delta_{i-1} T_{i-1} \otimes I k_{i}(x) \\
&-\sum_{i=1}^{N+1}(-1)^{i} \operatorname{tr} \Delta^{*} \delta_{i-1}^{\prime} T_{i-1} \otimes I k_{i}(x) \\
&= \sum_{i=1}^{N}(-1)^{i}\left[\operatorname{tr} \Delta^{*} \delta_{i-1} T_{i-1} \otimes I k_{i}(x)\right. \\
&\left.-\operatorname{tr} \Delta^{*} \delta_{i-1}^{\prime} T_{i-1} \otimes I k_{i}(x)\right] .
\end{aligned}
$$

Or l'expression entre crochets dans cette dernière sommation, est égale à

$$
\left[d \sum_{j=1}^{l} i_{X_{j}} \operatorname{tr} \Delta^{*} \sigma_{i-1}\left(\xi_{j}\right) \circ T_{i-1} \otimes I k_{i}\right](x)
$$

(cf. lemme 2.7); donc sur $W-C(f, G)$ on a

$$
-\sum_{i=0}^{N}(-1)^{i} \operatorname{tr} \Delta^{*} T_{i} \otimes I h_{i}=d \sum_{i=1}^{N} \sum_{j=1}^{l}(-1)^{i} i_{X_{j}} \operatorname{tr} \Delta^{*} \sigma_{i-1}\left(\xi_{j}\right) \circ T_{i-1} \otimes I k_{i} .
$$

Et en posant

$$
\begin{aligned}
& \bar{\Psi}=-\sum_{i=1}^{N} \sum_{j=1}^{l}(-1)^{i} i_{X_{j}} \operatorname{tr} \Delta^{*} \sigma_{i-1}\left(\xi_{j}\right) \circ T_{i-1} \otimes I k_{i} \\
& \bar{\Phi}=\sum_{i=0}^{N}(-1)^{i} \operatorname{tr} \Delta^{*} T_{i} \otimes I h_{i},
\end{aligned}
$$


on a bien

$$
\bar{\Psi} \in \Omega^{k-1}(W-C(f, G)), \bar{\Phi} \in \Omega^{k}(W), d \bar{\Psi}=\bar{\Phi} \operatorname{sur} W-C(f, G)
$$

et

$$
\int_{W} \bar{\Phi}=L_{G}(T)
$$

En effet

$$
\begin{aligned}
\int_{W} \bar{\Phi} & =\sum_{i=1}^{N} \int_{W} \operatorname{tr} \Delta^{*} T_{i} \otimes I h_{i} \\
& =\sum_{i=1}^{N}(-1)^{i} \int_{W} \sum_{\lambda_{n}^{i}=0}\left\langle T_{i} s_{n}^{i} \mid s_{n}^{i}\right\rangle \omega \\
& =\sum_{i=0}^{N}(-1)^{i} \sum_{\lambda_{n}^{i}=0}\left\langle T_{i} s_{n}^{i}, s_{n}^{i}\right\rangle \\
& =\sum_{i=0}^{N}(-1)^{i} \operatorname{Tr} H_{G}^{i}(T) \\
& =L_{G}(T) .
\end{aligned}
$$

Corollaire 2.10. - Si l'application $f$ ne possède pas de point de coincidence avec $G$, alors $L_{G}(T)=0$.

\section{Retour aux feuilletages}

Soit $\mathcal{F}$ un feuilletage riemannien transversalement orienté de dimension $p$ et de codimension $q$ sur une variété $M$ compacte connexe orientée de dimension $p+q$. On supposera que $M$ est munie d'une métrique riemannienne quasi-fibrée pour laquelle toutes les feuilles de $\mathcal{F}$ sont des sousvariétés minimales; une telle métrique existe si et seulement si $H^{q}(M / \mathcal{F}) \neq$ 0 (cf. [Ma]). Le feuilletage $\mathcal{F}$ sera orienté de telle façon que les orientations de $\mathcal{F}$ et de $\nu \mathcal{F}$ induisent l'orientation considérée sur $M$. On désignera par $\nu \in \Omega^{q}(M / \mathcal{F})$ la forme volume basique de $\mathcal{F}$ et par $\chi_{\mathcal{F}} \in \Omega^{p}(M)$ la forme caractéristique de $\mathcal{F}$ qui est donnée par $\chi \mathcal{F}=* \nu$ où $*: \Omega^{q}(M) \longrightarrow \Omega^{p}(M)$ est l'opérateur de Hodge associé à la métrique riemannienne sur $M$. Il est 
démontré dans $[\mathrm{Ru}]$ que la condition de minimalité pour les feuilles de $\mathcal{F}$ est équivalente à

$$
i_{X_{1}} \ldots i_{X_{p}} d \chi_{\mathcal{F}}=0, \quad \forall X_{1}, \ldots, X_{p} \in \Gamma(\mathcal{F}) .
$$

Si $\rho: M^{\sharp} \longrightarrow M$ est le $\mathrm{SO}(q)$-fibré principal des repères orthonormés directs de $\nu \mathcal{F}$ et $\mathcal{F}^{\sharp}$ le feuilletage relevé de $\mathcal{F}$ qui est T.P. de codimension $q+n$ sur $M^{\sharp}(n=q(q-1) / 2$ étant la dimension de $\mathrm{SO}(q))$ dont la fibration basique est notée $\pi: M^{\sharp} \longrightarrow W$; la structure riemannienne sur $\nu \mathcal{F}^{\sharp}$ définit une métrique riemannienne $\mathrm{SO}(q)$-invariante sur $W$; de plus si $\Theta$ désigne la forme différentielle basique et fermée sur $M^{\sharp}$ induisant la forme volume basique sur chaque fibre de $\pi$ (cf. [Ek], on peut supposer que $W$ est orientée de telle façon que si $\omega$ est la forme volume de $W$, la forme $\pi^{*}(\omega) \wedge \Theta$ et la forme volume basique $\nu^{\sharp}$ de $\mathcal{F}^{\sharp}$ définissent la même orientation transverse pour $\mathcal{F}^{\sharp}$; dans ces conditions si la fibration $\pi: M^{\sharp} \longrightarrow W$ est orientée par la forme $(-1)^{p n} \Theta \wedge \rho^{*}\left(\chi_{\mathcal{F}}\right)$, les orientations produit locales définies sur $M^{\sharp}$ par les fibrations orientées $\rho$ et $\pi$ seront les mêmes.

Considèrons maintenant une application de classe $C^{\infty}, f: M \longrightarrow M$ qui respecte le feuilletage $\mathcal{F}$, un $\mathcal{F}$-fibré hermitien $E$ sur $M$ et un morphisme de $\mathcal{F}$-fibrés $\varphi: f^{*} E \longrightarrow E$; le fibré $f^{*} E$ étant muni de la structure de $\mathcal{F}$-fibré hermitien image réciproque par $f$ de celle de $E$. On note $T$ l'endomorphisme de $\Gamma(E / \mathcal{F})$ qui à une section basique $s$ de $E$ associe la section basique $T(s)$ de $E$ définie par

$$
T(s)(x)=\varphi[x, s \circ f(x)] \quad \text { pour tout } x \in M .
$$

Si $\mathcal{F}$ est T.P. de fibration basique $\pi: M \longrightarrow W$ alors on a le diagramme commutatif

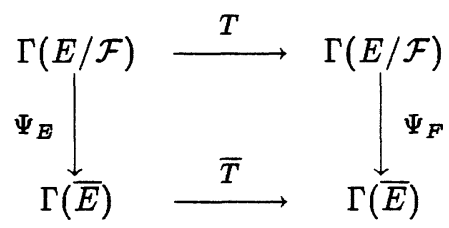

où $\bar{T}=\bar{\varphi} \circ \bar{f}^{*} ; \bar{f}: W \longrightarrow W$ étant l'application $C^{\infty}$ induite par $f$ et $\bar{\varphi}: \bar{f}^{*} \bar{E} \longrightarrow \bar{E}$ le morphisme de fibrés vectoriels défini par

$$
\begin{aligned}
& {[\bar{\varphi}(w, \alpha)](x)=\varphi[x, \alpha \circ f(x)],} \\
& \forall w \in W, \forall \alpha \in \bar{E}_{\bar{f}(w)}, \forall x \in \pi^{-1}(w) .
\end{aligned}
$$


Si $\mathcal{F}$ n'est pas nécessairement T.P., on suppose que $f: M \longrightarrow M$ est une application telle que pour tout $x \in M$ l'application $\nu_{x} f: \nu_{x} \mathcal{F} \longrightarrow \nu_{f(x)} \mathcal{F}$ induite par $d f_{x}$ est un isomorphisme qui préserve les orientations. Dans ce cas $f$ se relève en une application $f^{\sharp}: M^{\sharp} \longrightarrow M^{\sharp}$ qui respecte $\mathcal{F}^{\sharp}$ et qui induit une application de classe $C^{\infty} \bar{f}: W \longrightarrow W$; le morphisme $\varphi$ induit un morphisme de $\mathcal{F}^{\sharp}$-fibrés vectoriels

$$
\varphi^{\sharp}:\left(f^{\sharp}\right)^{*} E^{\sharp} \longrightarrow E^{\sharp}
$$

de la manière suivante : pour un élément $\left(z_{x}, f^{\sharp}\left(z_{x}\right), u_{f(x)}\right)$ dans la fibre de $\left(f^{\sharp}\right)^{*} E^{\sharp}$ au-dessus d'un repère transverse $z_{x} \in \rho^{-1}(x)$ en un point $x \in M$, on pose

$$
\varphi^{\sharp}\left(z_{x}, f^{\sharp}\left(z_{x}\right), u_{f(x)}\right)=\left(z_{x}, \varphi_{x}\left(u_{f(x)}\right)\right) .
$$

Proposition 3.1.- L'endomorphisme $T^{\sharp}$ de $\Gamma\left(E^{\sharp} / \mathcal{F}^{\sharp}\right)$ défini comme ci-dessus à partir de $f^{\sharp}$ et de $\varphi^{\sharp}$, préserve les sections basiques $\operatorname{SO}(q)$ invariantes (même si $f^{\sharp} n^{\prime} e s t$ pas équivariante).

Démonstration. - Si $s$ est un élément de $\Gamma_{\mathrm{SO}(q)}\left(E^{\sharp} / \mathcal{F}^{\sharp}\right)$ on peut écrire

$$
s(z)=(z, \alpha(z)), \quad \forall z \in M^{\sharp}
$$

où $\alpha$ est une application de $M^{\sharp}$ dans $E$ vérifiant :

$$
\alpha\left(z_{x}\right) \in E_{x}, \quad \alpha\left(z_{x} \cdot g\right)=\alpha\left(z_{x}\right), \forall x \in M, z_{x} \in \rho^{-1}(x), g \in \operatorname{SO}(q)
$$

On a donc :

$$
\begin{aligned}
{\left[T^{\sharp}(s)\left(z_{x} \cdot g^{-1}\right)\right] \cdot g } & =\left[\varphi^{\sharp}\left(z_{x} \cdot g^{-1}, f^{\sharp}\left(z_{x} \cdot g^{-1}\right), \alpha \circ f^{\sharp}\left(z_{x} \cdot g^{-1}\right)\right)\right] \cdot g \\
& =\left(z_{x} \cdot g^{-1}, \varphi_{x}\left[\alpha \circ f^{\sharp}\left(z_{x} \cdot g^{-1}\right)\right]\right) \cdot g \\
& =\left(z_{x}, \varphi_{x}\left[\alpha \circ f^{\sharp}\left(z_{x} \cdot g^{-1}\right)\right]\right) .
\end{aligned}
$$

Or nous avons

$$
\rho\left[f^{\sharp}\left(z_{x} \cdot g^{-1}\right)\right]=\rho\left[f^{\sharp}\left(z_{x}\right)\right]=f(x) .
$$


Il existe donc un élément $g_{0} \in \mathrm{SO}(q)$ tel que

$$
f^{\sharp}\left(z_{x} \cdot g^{-1}\right)=f^{\sharp}\left(z_{x}\right) \cdot g_{0}
$$

d'où

$$
\alpha\left[f^{\sharp}\left(z_{x} \cdot g^{-1}\right)\right]=\alpha\left[f^{\sharp}\left(z_{x}\right)\right]
$$

et par suite $T^{\sharp}(s)$ est invariante.

Dans ce cas, l'endomorphisme $\bar{T}_{\mathrm{SO}(q)}$, défini comme dans la section 2, n'est rien d'autre que la restriction de $\overline{T^{\sharp}}$ à l'espace des sections invariantes. On a donc le diagramme commutatif:

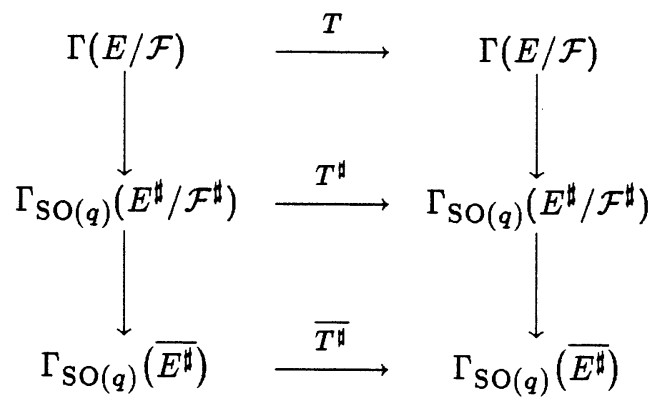

Considèrons maintenant un complexe transversalement elliptique

$$
(\mathcal{E}, d)=\left(\Gamma\left(E_{i} / \mathcal{F}\right), d_{i}\right)_{0 \leq i \leq N}
$$

avec un endomorphisme géométrique $T=\left(T_{i}\right)_{0 \leq i \leq N}$ défini à l'aide de l'application $f$ et d'une famille de morphismes de $\mathcal{F}$-fibrés (induisant l'identité sur $M$ )

$$
\varphi_{i}: f^{*} E_{i} \rightarrow E_{i}, \quad i=0, \ldots, N \text {. }
$$

Dans ce cas les applications linéaires

$$
\overline{T_{i}^{\sharp}}: \Gamma\left(\overline{E_{i}^{\sharp}}\right) \rightarrow \Gamma\left(\overline{E_{i}^{\sharp}}\right)
$$

forment un endomorphisme $\overline{T^{\sharp}}$ du SO $(q)$-complexe transversalement elliptique

$$
\begin{aligned}
\left(\overline{\mathcal{E}^{\sharp}}, \overline{d^{\sharp}}\right)= & \left(\Gamma\left(\overline{E_{i}^{\sharp}}\right), \overline{d_{i}^{\sharp}}\right)_{0 \leq i \leq N} \\
& -128-
\end{aligned}
$$


tel que le diagramme (dont les flèches verticales sont des isomorphismes)

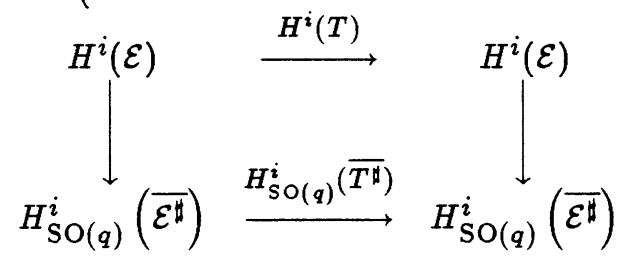

commute; d'où la proposition 3.3.

Proposition 3.3. - On a $L_{\mathcal{F}}(T)=L_{\mathrm{SO}(q)}\left(\overline{T^{\sharp}}\right)$.

Notons $C_{f}$ l'ensemble des points $x \in M$ tels que $x$ et $f(x)$ appartiennent à une même adhérence de feuille de $\mathcal{F}$; on vérifie aisément que $C_{f}$ n'est autre que le compact $\rho\left\{\pi^{-1}[C(\bar{f}, \mathrm{SO}(q))]\right\}$ de $M$.

THÉORÈME 3.4. - Il existe une ( $q-1)$-forme basique sur $M-C_{f}$ telle que $d \Psi$ se prolonge en une forme différentielle globale $\Phi \in \Omega^{q}(M / \mathcal{F})$ vérifiant

$$
L_{\mathcal{F}}(T)=\int_{M} \Phi \wedge \chi_{\mathcal{F}}
$$

De plus $L_{\mathcal{F}}(T)$ ne dépend que de la classe de $\Phi$ dans $H^{q}(M / \mathcal{F})$.

Démonstration. - Il existe (cf. théorème 2.9 et proposition 3.3) des formes différentielles $\bar{\Psi} \in \Omega^{k-1}(W-C(\bar{f}, \mathrm{SO}(q)))$ et $\bar{\Phi} \in \Omega^{k}(W)$ telles que

$$
d \bar{\Psi}=\bar{\Phi} \operatorname{sur} W-C(\bar{f}, \operatorname{SO}(q)) \quad \text { et } \quad \int_{W} \bar{\Phi}=L_{\mathrm{SO}(q)}\left(\overline{T^{\sharp}}\right)=L_{\mathcal{F}}(T) .
$$

Posons

$$
\Psi=\oint_{\rho} \pi^{*}(\bar{\Psi}) \wedge \Theta \quad \text { et } \quad \Phi=\oint_{\rho} \pi^{*}(\bar{\Phi}) \wedge \Theta
$$

(où $\oint$ désigne l'intégration suivant la fibre et $\Theta$ la forme fermée sur $M^{\sharp}$ induisant la forme volume basique sur chaque adhérence de feuille de $\mathcal{F}^{\sharp}$ ). Alors sur $M-C_{f}$, on a d'une part,

$$
\begin{aligned}
d \Psi & =d \oint_{\rho} \pi^{*}(\bar{\Psi}) \wedge \Theta \\
& =\oint_{\rho} \pi^{*}(\bar{\Phi}) \wedge \Theta \\
& =\Phi
\end{aligned}
$$


et, d'autre part,

$$
\begin{aligned}
\int_{M} \Phi \wedge \chi_{\mathcal{F}} & =\int_{M}\left(\oint_{\rho} \pi^{*}(\bar{\Phi}) \wedge \Theta\right) \wedge \chi_{\mathcal{F}} \\
& =(-1)^{p n} \int_{M^{*}} \pi^{*}(\bar{\Phi}) \wedge \Theta \wedge \rho^{*}\left(\chi_{\mathcal{F}}\right) \\
& =(-1)^{p n} \int_{W} \bar{\Phi} \wedge \oint_{\pi} \Theta \wedge \rho^{*}\left(\chi_{\mathcal{F}}\right) \\
& =\int_{W} \bar{\Phi} \\
& =L_{\mathcal{F}}(T) .
\end{aligned}
$$

Une conséquence immédiate de ce théorème est le corollaire 3.5 .

Corollaire 3.5. - Si $f$ ne fixe (globalement) aucune adhérence de feuille de $\mathcal{F}$ alors $L_{\mathcal{F}}(T)=0$.

Dans le cas particulier de l'endomorphisme identité du complexe différentiel $\left(\Omega^{*}(M / \mathcal{F}), d\right)$ des formes basiques sur $M$, nous avons une classe de cohomologie basique $[\Phi]$ dans $H^{q}(M / \mathcal{F})$ vérifiant

$$
\int_{M} \Phi \wedge \chi_{\mathcal{F}}=\sum_{i=0}^{q}(-1)^{i} \operatorname{dim} H^{i}(M / \mathcal{F})=\chi(M / \mathcal{F})
$$

De plus $[\Phi]$ coïncide avec la classe d'Euler de $M$ si $\mathcal{F}$ est le feuilletage par points. Ceci justifie donc la définition 3.6.

DÉfinition 3.6. - La classe $[\Phi]$ de $H^{q}(M / \mathcal{F})$ associée par le théorème 3.3 à l'endomorphisme identité du complexe des formes basiques sur $M$, sera appelée classe d'Euler basique de $(M, \mathcal{F})$.

Ce travail m'a été proposé par A. El Kacimi. Tout le long de son élaboration il n'a cessé de me guider et me prodiguer conseil. Je l'en remercie. 
Nombre de Lefschetz basique pour un feuilletage riemannien

\section{Références}

[At] Атгун (M.F.).- Elliptic operators and compact groups, Lecture Notes in Math., 401 (1974).

[AB] Atryah (M.F.) and Botт (R.) .- A Lefschetz fixed point formula for elliptic complexes, I, II, Ann. of Math., 86 (1967) pp.347-407; 88 (1968) pp. 451-491.

[Ek] El Kacimi Alaoui (A.) .- Opérateurs transversalement elliptiques sur un feuilletage riemannien et applications, Composito mathematica, 73 (1990) pp. 57-106.

[GM] Goresky (M.) and MCPherson (R.) .- Lefschetz fixed point formula on singular spaces, Conference au Congrès Singularités, Lille, (juin 1991).

[KT] Kamber (F.) and TONDEUR (P.) .- Foliated bundles and characteristic classes, Lecture Notes in Maths Springer-Verlag, 493 (1975).

[Ma] MASA (J.) . - Duality and minimality in Riemannian foliations, Preprint Universidad de Santiago de Compostela (1990).

[Mo] Molino (P.) . - Riemannian foliations, Progress in Math., 73 (1988).

[Ru] RUMMLER .- Quelques notions simples en géometrie riemannienne et leurs applications aux feuilletages compacts, Comment. Math. Helvetici, 54 (1979) pp. 224-239.

[To] Toledo (D.) .- On the Atiyah-Bott formula for isolated fixed points, J. differential geometry, 8 (1973) pp. 401-436. 